\title{
Tamanho de amostra para caracterização morfológica de frutos de pimenteira
}

\author{
Anderson Rodrigo da Silva ${ }^{1}$; Elizanilda R do Rêgo ${ }^{2}$; Paulo Roberto Cecon ${ }^{1}$ \\ ${ }^{1}$ UFV-CCE, Dep ${ }^{\text {to }}$ Estatística, 36570-000 Viçosa-MG; ${ }^{2}$ UFPB-CCA, Dep ${ }^{\text {to }}$ Ciências Fundamentais e Sociais, C. Postal 02, 58397-000, \\ Areia-PB; anderson.rodrigo@ufv.br
}

\section{RESUMO}

O objetivo deste estudo foi determinar o tamanho apropriado de amostra por meio da técnica de simulação de subamostras para a caracterização de variáveis morfológicas de frutos de oito acessos (variedades) de quatro espécies de pimenteira (Capsicum spp.), que foram cultivadas em área experimental da UFPB. Foram analisados tamanhos reduzidos de amostras, variando de 3 a 29 frutos, com 100 amostras para cada tamanho simulado em um processo de amostragem com reposição de dados. Realizou-se análise de variância para os números mínimos de frutos por amostra que representasse a amostra de referência (30 frutos) em cada variável estudada, constituindo um delineamento experimental inteiramente casualizado com duas repetições, onde cada dado representou o primeiro número de frutos na amostra simulada que não apresentou nenhum valor fora do intervalo de confiança da amostra de referência e que assim manteve-se até a última subamostra da simulação. A técnica de simulação utilizada permitiu obter, com a mesma precisão da amostra de 30 frutos, reduções do tamanho amostral em torno de $50 \%$, dependendo da variável morfológica, não havendo diferenças entre os acessos.

\begin{abstract} fruits

Sample size for morphological characterization of pepper

The appropriate sample size for the evaluation of morphological fruit traits of pepper was evaluated through a technique of simulation of subsamples. The treatments consisted of eight accessions of four pepper species (Capsicum spp.), cultivated in an experimental area of the Universidade Federal da Paraíba. Small samples, ranging from 3 to 29 fruits were evaluated. For each sample size, 100 subsamples were simulated with data replacement. The data were submitted to analysis of variance, in a complete randomized design, for the minimum number of fruits per sample. Each collected data consisted of the first number of fruits in the simulated sample without values out of the confidence interval. This procedure was done up to the last subsample simulation. The simulation technique provided reductions in the sample size, around 50\%, with the same accuracy of the sample of 30 fruits and the accessions did not differ significantly.
\end{abstract}

Keywords: Capsicum spp, subsamples, simulation.

\section{(Recebido para publicação em 10 de junho de 2010; aceito em 18 de janeiro de 2011) (Received on June 10, 2010; accepted on January 18, 2011)}

\begin{abstract}
$\mathrm{A}^{\mathrm{n}}$ produção de pimentas cresceu muito nos últimos anos, sendo utilizadas como condimento, nas indústrias farmacêutica e bélica, e como plantas ornamentais. Além de consumidas in natura, as pimentas podem ser processadas e utilizadas em diversos produtos alimentícios (Pinto et al., 2007).

As pimentas pertencem à família Solanaceae e ao gênero Capsicum. Este gênero possui de 20 a 25 espécies, normalmente classificadas de acordo com o nível de domesticação. As espécies e variedades domesticadas e semidomesticadas podem ser discriminadas por características morfológicas visualizadas principalmente nas flores e nos frutos. A grande variabilidade morfológica apresentada pelos frutos é destacada pelas múltiplas formas, tamanhos e colorações. $\mathrm{O}$ formato varia entre as espécies e dentro delas, existindo frutos alongados, arredondados, triangulares
\end{abstract}

ou cônicos, campanulados, quadrados ou retangulares (Carvalho \& Bianchetti, 2007)

$\mathrm{Na}$ comercialização dos frutos há determinadas classificações que são utilizadas para definir os preços de mercado; daí a importância de se caracterizar os frutos de pimenta, necessitando-se, para isso, de amostras confiáveis, pois, como afirma Callegari-Jaques (2003), o tempo necessário para estudar toda a população, as despesas e o número de pessoas envolvidas são de tal monta que tornam o estudo proibitivo.

É muito importante que a amostra retirada forneça uma representação precisa da população da qual ela é selecionada. Do contrário, as conclusões sobre a população podem ser distorcidas ou viesadas (Pagano \& Gauvreau, 2006). Todavia, na utilização da amostragem os resultados estão sujeitos a certo grau de incerteza, pois os dados mensurados em amostras podem conduzir a uma variação aleatória relativa ao método de medição, ao próprio material e também por considerar apenas uma parte da população (Heath, 1981).

Uma opção para se obter amostras de tamanho eficiente em estimar parâmetros populacionais desejáveis é a utilização da técnica de reamostragem de subamostras de tamanho reduzido ao de uma amostra de referência. Como afirmam Leite et al. (2009), o método da reamostragem permite uma comparação eficiente dos efeitos do tamanho da amostra na estimação de parâmetros genéticos e fenotípicos. Neste sentido, a técnica da simulação de subamostras permite obter, na maioria das vezes, um número inferior de dados da amostra referencial que seja capaz de fornecer as mesmas estimativas desta última e com a mesma exatidão. Trata-se de um processo pelo qual se sorteiam tantos 
dados quanto forem desejados para compor amostras de tamanho inferior à amostra de referência, de modo que haja reposição ou não dos dados utilizados para a simulação. Assim, é possível diminuir tanto os custos com mão-deobra quanto o tempo necessário para a caracterização dos frutos sem abrir mão de amostras confiáveis.

Pelo fato de que atualmente são utilizados diversos tamanhos amostrais para caracterizações morfológicas de pimentas, sem, contudo basear-se em pesquisas específicas, o objetivo deste estudo foi determinar o tamanho apropriado de amostras por meio da técnica de simulação de subamostras para caracterização de variáveis morfológicas de frutos de oito acessos (variedades) pertencentes a quatro espécies de pimenteira, gênero Capsicum.

\section{MATERIAL E MÉTODOS}

O presente trabalho foi desenvolvido no Laboratório de Biotecnologia e Melhoramento de Hortaliças do Centro de Ciências Agrárias da Universidade Federal da Paraíba (UFPB-CCA), de junho de 2009 a fevereiro de 2010, utilizando-se de oito acessos (variedades) de quatro espécies de pimenteira, gênero Capsicum, com características de frutos morfologicamente diferentes. Foram estudadas as espécies Capsicum chinense (acessos 2, 12, 13, 15 e 74), C. annuum (acesso 14), C. baccattum (acesso 72) e C. frutescens (acesso 4), pertencentes ao Banco de Germoplasma de Hortaliças da UFPB-CCA.

As plantas foram semeadas em bandejas de poliestireno expandido com 128 células contendo substrato comercial. Após a emissão de seis folhas definitivas as plantas foram transplantadas para campo em delineamento inteiramente casualizado com duas repetições, sendo cada repetição constituída de cinco plantas. Foram coletados todos os frutos da parcela e então obtida, aleatoriamente, uma amostra de 30 frutos de cada acesso por repetição, totalizando uma amostra de 60 frutos por acesso. As análises foram realizadas com amostras de frutos de oito acessos de pimenteira com frutos morfologicamente diferentes. Os tratos culturais seguiram as recomendações feitas por Filgueira (2000).

Seis características morfológicas foram avaliadas: Peso médio do fruto (PMF), comprimento do pedúnculo (CP), comprimento do fruto $(\mathrm{CF})$, maior diâmetro do fruto (MADF), menor diâmetro do fruto (MEDF) e espessura do pericarpo (EP), em milímetros, medidos com um paquímetro digital, modelo Stainless Hardened, precisão 0,01 mm. O peso médio do fruto, em gramas, foi determinado por meio de pesagem em balança eletrônica Bioprecisa, modelo JA3003N.

Posteriormente, determinou-se o número mínimo de frutos por amostra que representou o acesso com precisão semelhante à amostra de referência. Utilizou-se o método de simulação, consistindo de reamostragens de subamostras, para as quais foram analisadas as médias aritméticas estimadas das características de frutos de cada acesso.

Foram analisados tamanhos de amostras reduzidos, variando de 3 a 29 frutos, com 100 amostragens para cada tamanho simulado em um processo de amostragem com reposição de dados. Por exemplo, para obter estimativa das médias da amostra de 3 frutos, o software utilizado realizou 100 sorteios consecutivos de 3 frutos na amostra original de 30 frutos e fez as estimativas a partir dos 100 valores obtidos. As estimativas de médias das características para cada tamanho de amostra analisado foram plotadas em gráfico para análise e visualização da estabilização de cada estimativa de acordo com o número de frutos de cada subamostra simulada, o que ocorre quando o tamanho das subamostras passa a representar adequadamente a amostra de referência. Considerou-se que a amostra de tamanho reduzido representou a amostra de referência quando não houve nenhum valor simulado fora do intervalo de confiança $\left(\mathrm{IC}_{95 \%}\right)$ para esta amostra, com probabilidade de 95\%.

Realizou-se análise de variância para os números mínimos de frutos por amostra que representasse a amostra de referência em cada variável estudada, constituindo um delineamento experimental inteiramente casualizado, com duas repetições, onde cada dado repre- sentou o primeiro número de frutos na amostra simulada que não apresentou nenhum valor de média estimada fora do $\mathrm{IC}_{95 \%}$, construído para a respectiva amostra de referência de cada acesso, e que assim manteve-se até a última subamostra da simulação. Para isto, os dados foram transformados em $(\mathrm{x}+0,5)^{1 / 2}$, segundo metodologia proposta por Gomes (1987). As simulações e a análise de variância foram realizadas utilizandose o programa GENES, versão versão 2006.4.1 (Cruz, 2001).

\section{RESULTADOS E DISCUSSÃO}

Pelo resumo da análise de variância (Tabela 2), observa-se que não houve efeito significativo entre os acessos, pelo teste $\mathrm{F}$ a 5\% de significância, para quaisquer das variáveis estudadas. Tal fato indica que o menor número de frutos por amostra simulada que não apresentou valores de média fora do intervalo de confiança da respectiva amostra de referência, é suficiente para que se efetue a caracterização morfológica dos frutos de quaisquer dos acessos estudados, conferindo ganho de tempo e economia de mão-de-obra. Essa afirmativa é válida para quaisquer das variáveis morfológicas em estudo, desde que se observe qual é o menor número de frutos por amostra simulada sempre que se alterar a variável, independente do acesso (Tabela 1).

$\mathrm{Na}$ Tabela 1 é possível observar os números mínimos de frutos por amostra simulada para os diferentes acessos de pimenteira em todas as variáveis estudadas, onde nenhuma média se situou fora do $\mathrm{IC}_{95 \%}$ (das amostras de 30 frutos) construído para cada variável em cada acesso. Neste caso, entende-se que, para a variável peso médio dos frutos, por exemplo, amostras com 16 frutos são suficientes para a determinação desta característica com a mesma exatidão das amostras iniciais de 30 frutos, com uma margem de erro inferior a 5\%. Isso implica em uma redução de 46,7\% dos gastos com caracterização para esta variável.

Para as outras variáveis tem-se $\mathrm{CP}=15, \mathrm{CF}=14, \mathrm{MADF}=15, \mathrm{MEDF}=$ 14 e $\mathrm{EP}=14$ frutos por amostra como sendo suficientes para a caracterização 
Tabela 1. Estimativas do número mínimo de frutos por amostra simulada para caracterização de cada variável morfológica em frutos de oito acessos (variedades) de pimenteira (estimation of the least number of fruits per simulated sample for the characterization of eight pepper accessions (varieties)). Areia, UFPB, 2009-2010.

\begin{tabular}{lcccccccc}
\hline Acesso & PMF & CP & CF & MADF & MEDF & EP & Valor médio & Maior valor \\
\hline 2 & 18 & 17 & 17 & 18 & 18 & 16 & 17,33 & 18 \\
12 & 16 & 17 & 18 & 20 & 15 & 15 & 16,83 & 20 \\
13 & 21 & 15 & 16 & 19 & 17 & 18 & 17,67 & 21 \\
15 & 18 & 16 & 18 & 18 & 16 & 14 & 16,67 & 18 \\
74 & 18 & 18 & 19 & 15 & 19 & 20 & 18,16 & 20 \\
14 & 18 & 18 & 15 & 18 & 15 & 17 & 16,83 & 18 \\
72 & 19 & 18 & 14 & 20 & 18 & 20 & 18,16 & 20 \\
4 & 20 & 17 & 19 & 22 & 14 & 18 & 18,33 & 22 \\
\hline Valor médio & 18,5 & 17 & 17 & 18,75 & 16,5 & 17,25 & -- & -- \\
\hline Menor valor & 16 & 15 & 14 & 15 & 14 & 14 & -- & -- \\
\hline
\end{tabular}

$\mathrm{PMF}=$ peso médio do fruto; $\mathrm{CP}$ comprimento do pedúnculo; $\mathrm{CF}=$ Comprimento do fruto; MADF e MEDF= maior e menor diâmetro do fruto, respectivamente; $\mathrm{EP}=$ espessura do pericarpo $(\mathrm{PMF}=$ average fruit weight; $\mathrm{CP}=$ pedicel length; $\mathrm{CF}=$ fruit length; $\mathrm{MADF}$ and $\mathrm{MEDF}=$ the largest and the smallest fruit diameter, respectively; $\mathrm{EP}=$ pericarp thickness).

Tabela 2. Resumo da análise de variância ${ }^{1}$ para as estimativas dos números mínimos de frutos que representam a amostra de referência (30 frutos) em cada variável estudada (analysis of variance ${ }^{1}$ for the estimation of the least number of fruits which represents the reference sample (30 fruits) in each evaluated trait). Areia, UFPB, 2009-2010.

\begin{tabular}{lcccccccc}
\hline \multirow{2}{*}{ Fonte de variação } & \multirow{2}{*}{ GL } & \multicolumn{5}{c}{ Quadrados médios } \\
\cline { 3 - 8 } & & PMF & CP & CF & MADF & MEDF & EP \\
\hline Acessos & 7 & $0,060^{\mathrm{NS}}$ & $0,039^{\mathrm{NS}}$ & $0,082^{\mathrm{NS}}$ & $0,099^{\mathrm{NS}}$ & $0,085^{\mathrm{NS}}$ & $0,145^{\mathrm{NS}}$ \\
Resíduo & 8 & 0,071 & 0,101 & 0,064 & 0,052 & 0,072 & 0,069 \\
\hline CV $(\%)$ & -- & 6,22 & 7,67 & 6,13 & 5,27 & 6,63 & 6,34 \\
\hline
\end{tabular}

$\mathrm{PMF}=$ peso médio do fruto; $\mathrm{CP}=$ comprimento do pedúnculo; $\mathrm{CF}=$ comprimento do fruto; MADF e MEDF= maior e menor diâmetro do fruto, respectivamente; $\mathrm{EP}=$ espessura do pericarpo; ${ }^{\mathrm{NS}}=$ não significativo a $5 \%$ de significância pelo teste $\mathrm{F}$; ${ }^{1}$ dados transformados por $(\mathrm{x}+0,5)^{1 / 2}(\mathrm{PMF}=$ average fruit weight; $\mathrm{CP}=$ pedicel length; $\mathrm{CF}=$ fruit length; $\mathrm{MADF}$ and $\mathrm{MEDF}=$ the greatest and the smallest fruit diameter, respectively; $\mathrm{EP}=$ pericarp thickness; ${ }^{\mathrm{NS}}$ not significant at $5 \%$ significance by $\mathrm{F}$ test; ${ }^{1}$ data processed by $\left.(\mathrm{x}+0,5)^{1 / 2}\right)$.

morfológica dos frutos dos acessos de pimenteira em estudo. Conforme relataram Zanon \& Storck (2000) em estudo parecido, números superiores a estes não acrescentariam maiores informações e não proporcionariam redução significativa do erro.

$\mathrm{Na}$ Figura 1, observa-se a dispersão das 100 estimativas para as médias das variáveis estudadas, em cada tamanho amostral simulado, observando-se uma tendência de estabilização destas estimativas dentro do intervalo de confiança $\left(\mathrm{IC}_{95 \%}\right)$ construído para a amostra de referência (30 frutos), onde se verifica que o número de médias estimadas fora do IC diminui constantemente a partir do momento em que se aumenta a quantidade de frutos na amostra, sendo que tende a se estabilizar a partir dos 15 frutos por amostra com nenhuma media fora do $\mathrm{IC}_{95 \%}$ construído para a amostra de referência de cada variável em cada acesso.

Silva et al. (2009) encontraram resultados parecidos quando realizaram simulação para avaliar o número mínimo de plantas por parcela a partir da estabilização da média de várias características de raízes de cenoura, tomando-se como representativo da população, o número mínimo de plantas por parcela obtido de uma série constante (de números de plantas por parcela) que estivesse dentro do limite do desvio padrão calculado com a amostra de referência (20 plantas por parcela), obtendo assim, reduções de até 50\% no número de plantas por parcela, dependendo da variável.

Lorentz et al. (2003), estimaram a utilização de aproximadamente 35 de um total de 60 plantas por fileira de pepino tipo conserva em estufa plástica para avaliação da massa fresca total dos frutos, com uma semi-amplitude da média de $20 \%$.

Henriques Neto et al. (2004) estudando o tamanho ótimo da parcela em experimentos com trigo sob as formas de plantio convencional e direto, constataram que os tamanhos de parcelas variando de 12 (para plantio convencional) e 24 (para plantio direto) a 96 unidades básicas apresentam variâncias estatisticamente iguais, não ocorrendo maior precisão experimental com o aumento do tamanho das parcelas.

Em experimentos com cana-deaçúcar, utilizando 20 plantas por parcela e com uma adaptação da técnica de reamostragem bootstrap, visando determinar o tamanho da amostra para avaliação de famílias de irmãos completos, Leite et al. (2009) também verificaram que as estimativas do tamanho da amostra 


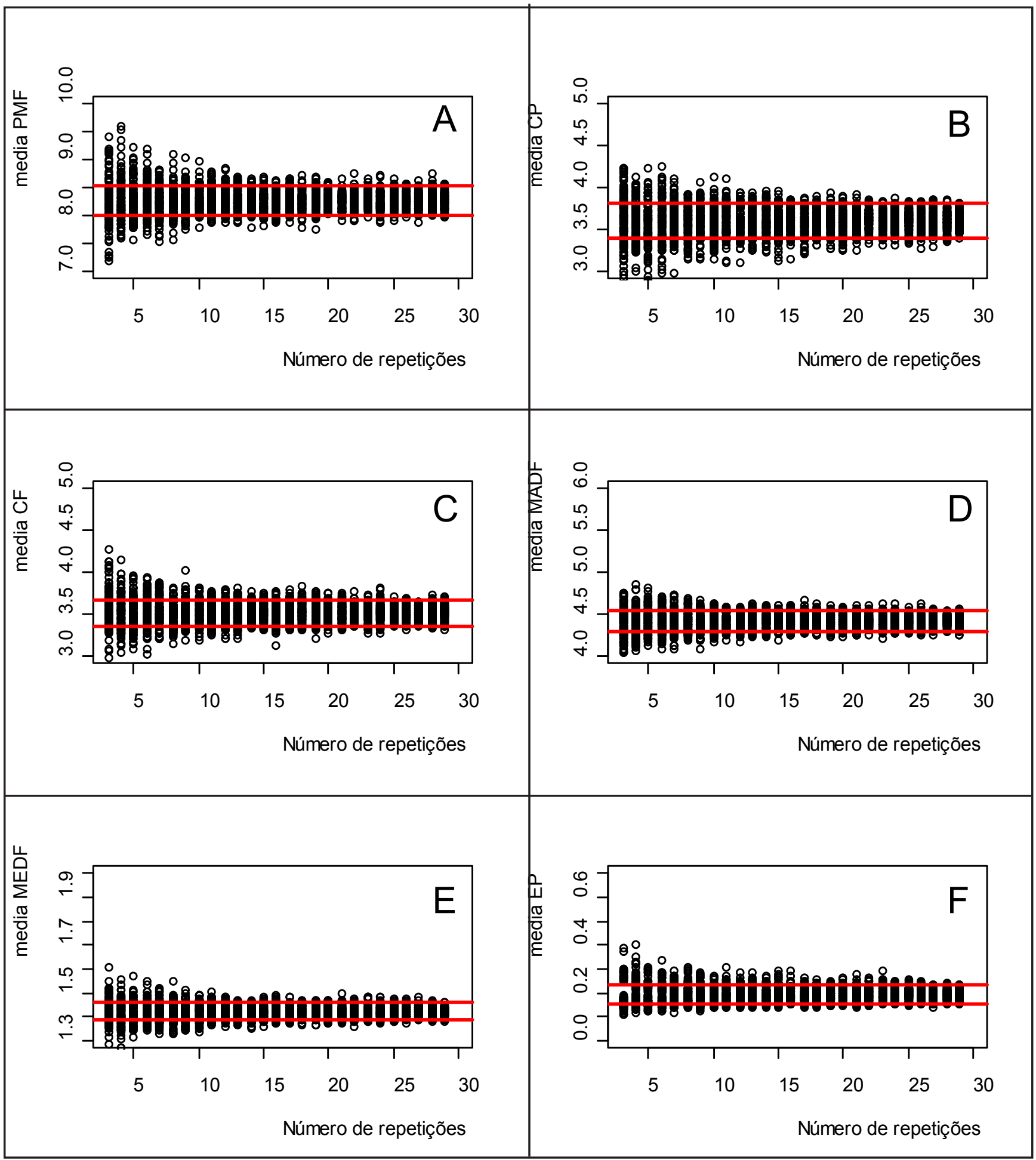

Figura 1. Dispersão das 100 estimativas de média de peso médio do fruto (A), comprimento do pedúnculo (B), comprimento do fruto (C), maior e menor diâmetro do fruto (D e E, respectivamente) e de espessura do pericarpo (F) de frutos de pimenteira em cada tamanho amostral simulado (dispersion of 100 estimates of average fruit weight (A), peduncle length (B) fruit length (C), the greatest and the smallest fruit diameter (D and E, respectively) and pericarp thickness (F) of pepper fruits in each simulated sample size). Areia, UFPB, $2009-2010$.

variaram de acordo com a variável em estudo e afirmaram a necessidade de 16 plantas por parcela (96 indivíduos por família) como sendo suficiente para obter estimativas fidedignas de todos os parâmetros avaliados em todas as variáveis estudadas.

Em caracteres de cultivares de feijão, Cargnelutti Filho et al. (2008) verificaram que os tamanhos de amostras devem ser de pelo menos 10 plantas por parcela experimental. Estudando tamanhos de amostras para experimentos com Eucalyptus salgna Smith, Zanon et al. (1997) verificaram a necessidade de 
8 a 26 plantas por unidade experimental. Já em pimenteiras do gênero Capsicum, as informações quanto ao tamanho apropriado de amostras para caracterização morfológica são escassas.

Tem-se portanto, que a técnica de simulação de amostras de tamanho reduzido permitiu obter, com a mesma exatidão da amostra de 30 frutos, reduções em torno de $50 \%$ quanto ao número de frutos de pimentas Capsicum para caracterização morfológica, dependendo da variável, sendo que todas as variáveis estudadas apresentaram significativa redução, podendo-se utilizar: $\mathrm{PMF}=16$, $\mathrm{CP}=15, \mathrm{CF}=14, \mathrm{MADF}=15, \mathrm{MEDF}=$ 14 e $\mathrm{EP}=14$ frutos por amostra como sendo suficientes para a caracterização destas variáveis morfológicas dos frutos de quaisquer dos acessos estudados.

\section{REFERÊNCIAS}

CALLEGARI-JAQUES SM. 2003. Bioestatística: princípios e aplicações. Porto Alegre: Artmed. $256 \mathrm{p}$.
CARGNELUTTI FILHO A; RIBEIRO ND; STORCK L; JOST E; POERSCH NL. 2008. Tamanho de amostra de caracteres de cultivares de feijão. Ciência Rural 38: 635-642.

CARVALHO SIC; BIANCHETTI LB. 2007. Pimenta (Capsicum spp.): Botânica. Embrapa Hortaliças. Brasília, Nov. 2007. Disponível em http://sistemasdeproducao.cnptia.embrapa.br/ FontesHTML/Pimenta/Pimenta_capsicum spp/botanica.html. Acessado em 19 de novembro de 2009.

CRUZ CD. 2001. Programa Genes versão Windows. Viçosa: UFV. 642p.

FILGUEIRA FAR. 2000. Novo Manual de Olericultura: Agrotecnologia moderna na produção e comercialização de hortaliças. Viçosa: UFV. 402p.

GOMES FP. 1987. A estatística moderna na pesquisa agropecuária. $3^{\mathrm{a}}$ ed. Piracicaba: POTAFOS. $162 \mathrm{p}$.

HEATH OVS. 1981. A estatística na pesquisa cientifica. São Paulo: USP. 95p.

HENRIQUES NETO D; SEDIYAMA T; SOUZA MA; CECON PR; YAMANAKA CH; SEDIYAMA MAN; VIANA AES. 2004. Tamanho de parcelas em experimentos com trigo irrigado sob plantio direto e convencional. Pesquisa Agropecuária Brasileira 39: $517-$ 524.

LEITE MSO; PETERNELLI LA; BARBOSA MHP; CECON PR; CRUZ CD. 2009. Sample size for full-sib family evaluation in sugarcane.
Pesquisa Agropecuária Brasileira 44: $1562-$ 1574.

LORENTZ LH; LÚCIO AD; BOLIGON AA; CARPES RH. 2003. Estimativas de parâmetros para planejamento de experimentos com pepino tipo conserva em estufa plástica em Santa Maria-RS. In: CONGRESSO BRASILEIRO DE OLERICULTURA, Anais... Recife: ABH. 4p.

PAGANO M; GAUVREAU K. 2006. Principios de bioestatística. Tradução da $2^{\mathrm{a}}$ edição Norteamericana. São Paulo: Thomson Learning. $506 \mathrm{p}$.

PINTO CMF; CALIMAN FRB; MOREIRA GR; MATTOS RN; ROCHA PRR; VENZON M; PAULA JÚNIOR TJ. Pimenta (Capsicum spp.). In: PAULA JÚNIOR TJ; VENZON M. (coord). 2007. 101 Culturas: manual de tecnologias agrícolas. Belo Horizonte: EPAMIG. p. 625-632.

SILVA GO; VIEIRA JV; VILLELA MS. 2009. Tamanho de amostra para avaliação de caracteres de cenoura em sistemas de cultivo agroecológico. Horticultura Brasileira 27: 166-170.

ZANON MLB; STORCK L. 2000. Tamanho ótimo de parcelas experimentais para Eucalyptus saligna Smith. em dois estádios de desenvolvimento. Cerne 6: 104-111.

ZANON MLB; STORCK L; FINGER CAG; HOPPE JM. 1997. Tamanho de amostra para experimentos de Eucalyptus salgna Smith em Viveiro. Ciência Florestal 7: 133-138. 